\title{
Le ton stendhal : postures, allures, textures
}

Philippe Jousset

\section{OpenEdition}

Journals

Édition électronique

URL : http://journals.openedition.org/recherchestravaux/343

DOI : 10.4000/recherchestravaux.343

ISSN : 1969-6434

\section{Éditeur}

UGA Éditions/Université Grenoble Alpes

\section{Édition imprimée}

Date de publication : 15 juillet 2009

Pagination : 5-21

ISBN : 978-2-84310-146-5

ISSN : 0151-1874

\section{Référence électronique}

Philippe Jousset, « Le ton stendhal : postures, allures, textures », Recherches \& Travaux [En ligne], 74 | 2009, mis en ligne le 28 février 2011, consulté le 08 septembre 2020. URL : http://

journals.openedition.org/recherchestravaux/343; DOI : https://doi.org/10.4000/recherchestravaux 343

(c) Recherches \& Travaux 


\section{Le ton stendhal: postures, allures, textures}

\section{Introduction}

Stendhal écrit comme il pense. Il a la langue de son génie. [...] de tous les hommes, il est le moins auteur. [...] Celui-là n'est pas homme de lettres.

André Suarès

Cette recherche sur le ton Stendhal se propose deux objectifs en un : le second, le plus général, serait de faire un peu de clarté sur un sujet que l'imprécision des définitions et la manière dont plusieurs corporations se disputent le terme, c'est-à-dire l'approprient à leurs fins, rendent assez confus, ou flou'. Mais le premier objectif, qui peut être une voie pour espérer atteindre le second, vise plus spécifiquement Stendhal. Comme cas particulier et exemplaire à la fois. Il existe en effet une prévention de beaucoup de commentateurs, parmi lesquels des esprits très estimables, à l'égard du style Stendhal : non pas qu'ils le jugent médiocre - quoique certains le censurent ${ }^{2}-$ mais ils doutent de bonne

I. Le titre de l'ouvrage de P. Soler, par exemple, réunit Genres, formes, tons (PUF, 200I) sans prendre le risque de définir précisément ces derniers, vite oubliés au profit des deux autres termes, traditionnellement mieux cernés. Il est simplement suggéré que le ton est une modalité plus permanente que le genre et peut lui survivre. Hostile à une conception binaire du style, É. Bordas explique cependant que le ton vient «doubler la signification de l'énoncé, pour laisser deviner la valeur pragmatique de l'énonciation (ton ironique, ton doucereux, ton menaçant, etc.). [...] Le ton est le premier niveau de présence du style, si style il y a [...]» («Style gay?», Littérature, $\mathrm{n}^{\circ}$ I47, Larousse, septembre 2007, p. I 20, repris et développé dans «Style». Un mot et des discours, Kimé, 2008, p. I74-I 84).

2. On pense avant tout à Balzac lecteur de La Chartreuse, et à la remarque de G. Blin : «Ce ton de Stendhal lui est à ce point inaliénable, qu'il éclate comme un reproche là où Balzac, 
foi que Stendhal possède véritablement un style’3. Les mêmes cependant lui accordent un ton, comme si celui-ci était un succédané de style - ou une sorte de style subtil, invisible, manquant de préméditation, de volonté, ou dénué de travail ? Le discours sur le naturel et le dilettantisme trouve à s'employer où la stylistique renonce. Il s'ensuit un glissement vers le beylisme, terme qui peut être entendu de diverses façons, mais dont on conçoit facilement qu'il vise, par-delà ou au travers des écrits stendhaliens, une certaine «philosophie de l'existence», une manière de regarder la vie «comme un bal masqué» (Pierre-Louis Rey).

On a dit confraternellement de Balzac qu'il avait un style défectueux et déplorable, que Flaubert «écrivait mal», etc., mais Stendhal, lui, manquerait donc tout simplement de style, et pour une raison un peu paradoxale : il possède une manière si personnelle que la sémiologie qui l'exprime n'atteindrait pas le seuil critique où il peut faire l'objet d'une étude réglée. Car chacun, par ailleurs, parfois pour le déplorer, lui reconnait un caractère propre. Certains, comme composante du style, d'autres, en lieu et place d'un style authentique. De fait, le ton est souvent négligé, traité comme parent pauvre du Style avec majuscule, et souffre d'une sous-entente théorique ${ }^{4}$.

Nous n'en appellerons dans cette introduction apéritive qu'à deux témoignages, mais parmi les plus représentatifs et les plus argumentés, ceux de Georges Blin et Paul Valéry.

Il n'est pas étonnant que celui qui, sur la plupart des aspects de la poétique stendhalienne, a ouvert parmi les voies les plus fécondes, soit aussi l'un de ceux qui a le mieux posé cette question du ton. Dans les premières lignes de son Stendhal et lesproblèmes du roman, Georges Blin justifie la nécessité de poser lesdits

dans son grand article sur la Chartreuse au lieu de se borner à résumer, vient à citer. Si Balzac $[\ldots]$ nous restitue si mal la physionomie que nous connaissons à l'ouvrage [...] c'est avant tout parce que, le récrivant dans un style qui trahit son pathos à lui, il n'en conserve rien au point de vue du ton» (Stendhal et les problèmes du roman, José Corti, 1954, p. 320).

3. Un troisième moment, ou synthèse, se rencontre dans le jugement de Remy de Gourmont, par exemple : «Bien qu'au premier abord il semble n’écrire qu'assez mal, surtout pour ces hommes emplis de rhétorique qu'on appelle les lettrés, il n'en faut pas moins le considérer comme un des maitres du style» («Le style et l'art de Stendhal», Promenades littéraires, $5^{\mathrm{e}}$ série, Mercure de France, $9^{\mathrm{e}}$ éd., I923, p. 106).

4. L'école russe ou Cercle de Bakhtine forme une exception; l'importance du ton y est reconnue, et on y déplore qu'il soit «l'aspect le moins étudié de la vie verbale» (voir T. Todorov, Mikhaïl Bakbtine. Le Principe dialogique suivi de Écrits du Cercle de Bakbtine, Seuil, «Poétiques», I 98 I, p. 83). Chez Bakhtine, le ton (ou intonation, plus souvent) est responsable du sens global de l'énoncé, et étroitement lié à l'intention et à l'orientation sociale de l'énoncé : il commande au choix des mots et à la composition du matériau verbal, et il se définit comme réglage à l'égard de l'interlocuteur aussi bien que de l'objet de l'énoncé (ou «héros»). L'œuvre poétique, en définitive, consiste en «quelques déplacements d'intonations [...] sur le fond des anciennes évaluations, des anciennes intonations» (V. N. Volochinov, cité par Todorov, ibid., p. 77-78). 
problèmes en termes de personnalité par le fait que Stendhal, particulièrement à cause de ses légendaires interventions d'auteur's (et de narrateur - avec lui la frontière est toujours fluctuante, et c'est bien à la faveur de ce doute que la question du ton fait une entrée remarquée), peut être tenu pour le premier des personnages de ses fictions. Ce que Blin appelle ce «manège d'apologie», que souffrent les genres du journal, de la critique ou de l'autobiographie, Stendhal le transporte tel quel dans l'univers romanesque, et contribue ainsi à prolonger, ou revigorer, un certain type de roman qui brouille les partages reconnus entre les genres, ce que tout le XVIII ${ }^{\mathrm{e}}$ siècle en particulier avait pratiqué sans états d'âme.

On perçoit d'emblée que le ton Stendhal repose peut-être d'abord sur une forme de commerce entre le créateur et sa créature (et le lecteur, par ricochet) qui rend improbable leur distinction absolue et, accessoirement, heurte de front l'un des dogmes de la «modernité» en matière d'études littéraires- la nette séparation des figures de l'auteur, du narrateur et du personnage - au profit d'une comédie de masques et de simulacres intermédiaires. Chez lui, en effet, le narrateur se trouve toujours lié par son protagoniste, et le narrateur souvent compromis au narrateur. Le ton unifie d'une certaine façon ou parfond ce que le décret de la poétique distingue dans son premier article : tu ne confondras pas l'«homme» et son discours, l'individu et l'«œuvre». Le style de vie prêté aux personnages, la nature des propos que développent ses porte-parole,

[...] bref, le tempo de leur moralité, explique Georges Blin, trahissent un choix, d'ordre éthique, que ne peut manquer de contresigner celui qui, de lui-même, s'est désigné, et dans la plus forte acception du terme, comme l'auteur.

Il en résulte une sorte de transsubstantiation de la réalité et de la fiction:

Dans le vécu, comme Henri Beyle se vise sous les espèces d'un héros stendhalien, c'est l'imaginaire qui subvient au réel, et le soulève; dans les romans, tout au contraire, $[\ldots]$ c'est le réel - autrement dit sa subsistance personnelle, sa présence comme individu - qui commandite l'imaginaire. Bref, comme homme, il a été le romancier de lui-même, et comme romancier un auteur si peu apte à se renoncer comme homme. (p. 32I)

Le ton serait donc le sceau d'une imparfaite séparation entre auteur, narrateur et personnage, un défaut d'élaboration de distance fictionnelle, puissant facteur d'illusion, à laquelle le lecteur, quatrième instance, se trouve lui-même commis.

Georges Blin - et nous retrouvons partout cette assimilation, dont il faudrait interroger ou le bien-fondé ou l'abus - traite indifféremment du ton

5. G. Blin précise : ces interventions «ne fussent-elles que de ton»... 
sous son nom propre ou sous celui de voix ${ }^{6}$. Il attribue à son «entregent» la principale vertu de créer une «prise directe» du romancier sur son argument. En articulant les faits de manière neutre, «sans nul accent» (encore un mot nouveau et important à interroger), le romancier réussirait à se faire comprendre; par le ton, il se fait reconnaître:

La voix, c'est en effet, tout ce qui, de l'histoire contée, ressortit au phénoménal. [...] Elle seule est susceptible de conférer de l'existence au roman, lequel, sans sa médiation, resterait un bloc d'essence coupé du temps. Nous donnant à enregistrer des contingences de timbre, de ton et de débit, elle nous force à appréhender le romancier comme existant, autrement dit à nous installer en coexistence avec lui $[. .$.$] la voix, en teignant le conte aux couleurs de celui qui raconte, tend à$ l'office de garantir la fiction en garantissant le garant. (p. 317 )

Par cette collusion avec la temporalité de la lecture, le roman nous rend contemporain des personnages aussi bien que de l'auteur contant et retisse les fils qui unissent notre situation à la leur :

Le ton seul, en effet, est capable de nous remettre dans le vis-à-vis du narrateur, à son heure, et dans l'illusion que comme cela a lieu dans l'audition réelle où notre présent vient sans cesse correspondre au présent de notre informateur, ici notre lecture se développe en parfaite concomitance avec une successive écriture. (p. 318$)$

Certes, rectifie aussitôt Georges Blin, ce présent est un présent «de l'ordre du comme si», un présent de complaisance, pour ainsi dire, ou «de comédie», qui s'offre toutefois «avec le moindre coefficient possible d'idéalité». (p. 3 I 8 319)

Paul Valéry, dans un célèbre texte de Variété du particularisme stendhalien dans la perspective d'une étude du ton. De cette contribution très riche, qui est celle d'un écrivain, donc pas tout à fait «dans les règles » et qu'on ne saurait instrumentaliser aux fins de notre propre démonstration sans perdre de son cours et de ses nuances, nous ne voudrions extraire que des sentences, des semences de réflexion, sur ce qui touche au vif de la question, mettant l'accent sur ce qui nous apparait comme les points forts de son développement.

Le point de départ de Valéry est cette affirmation des plus nettes, souvent citée : «Ce qui frappe le plus dans une page de Stendhal, [...] c'est le Ton.»

6. L'étude de Fr. Coulont-Henderson, au titre/sous-titre parlant, Le Désir de la voix vive. Étude du «ton» chez. Stendhal (Birmingham, Summa Publications, I 990), dans son chapitre recensant les données lexicales, prend en compte cinq termes : «ton», «air», «accent», «nuance» et «voix». Pour M. Blanchot, «le ton n'est pas la voix de l'écrivain, mais l'intimité du silence qu'il impose à la parole» (L'Espace littéraire, Gallimard «Essais», I 95 5, p. 22).

7. P. Valéry, Variété, dans CEuvres, t. I, J. Hytier éd., Gallimard, «Bibliothèque de la Pléiade», 1957. 
D'accord avec le diagnostic de Blin, il soutient que chez Stendhal, l'homme domine l'écrivain, ou plutôt domine le narrateur - l'écrivain étant davantage un rôle, quand le narrateur n'est qu'une simple fonction. Le ton, pour Valéry, c'est donc l'habitation de l'écriture par la parole de quelqu'un. Une parole assez individuelle pour qu'on y entende «un homme». Et qui sache se rendre assez intéressant pour l'être plus que ce qu'il narre, ou, soyons précis : pour que ce qu'il narre n'ait d'intérêt que dans la mesure même où il est porté par le disant. La réussite du ton chez Stendhal consisterait à rendre entièrement solidaires le discours et celui qui le tient, à lier absolument le sort de ce qui est dit à celui qui le dit, à transfuser la personne dans l'écriture et, dans la même opération, puisqu'elle est à double sens, à «personnaliser», au sens fort, l'écriture : pas seulement à lui donner une marque personnelle (cela va de soi), mais à faire du texte une sorte d'individu (avec les prédicats qu'on porte généralement au compte de l'individu : qualités de présence, de vivant, de versatilité...).

Or, l’homme qui «représente» verse immanquablement dans le comédien. Ce qui est au cœur de la problématique, c'est la négociation infinie, aporétique, de la sincérité. Et l'écueil qui la menace pourrait se désigner du nom (que Valéry n'emploie pas) d'histrionisme : lorsque l'homme faitl'intéressant. Avant d'en venir à la viciation de cette qualité centrale de la prose stendhalienne, Valéry en détaille les moyens. L'objet principal du recueil d'études que nous proposons aujourd'hui étant d'explorer les aspects du ton, nous nous contenterons d'indiquer les limites générales à l'intérieur desquelles joue cette parole parlante, soit : l'empan du paradoxe du ton'.

La formule de Valéry du ton selon Stendhal est la suivante : «écrire comme on parle». Mais, quelques lignes plus loin, cette première formule n'est pas encore sèche qu'il la retouche ainsi : "écrire presque comme on se parle». Se sont ajoutés, dans ce repentir, le réfléchi et le dièse du presque, qui en est une conséquence. Car si on peut bien écrire comme on parle (appelons cela «oralité écrite $\left.{ }^{\mathrm{I}} \gg\right)$, Valéry sait bien que se parler à soi-même est une demi-

8. On lira ici même la contribution d'A. Novak-Lechevalier sur les rapports du théâtre et du roman dans la question du ton chez Stendhal.

9. Sur ce paradoxe, on lira notamment l'article de G. Kliebenstein ici même, particulièrement le début.

Io. «Le terme de "ton", souligne D. Maingueneau, présente l'avantage de valoir aussi bien à l'écrit qu'à l'oral : on peut parler du “ton” d'un livre.» («Éthos, scénographie, incorporation», dans R. Amossy éd., Image de soi dans le discours. La construction de l'éthos, Delachaux et Nietslé, I 999, p. 78-79). Le même auteur avait excellemment posé le problème dans un ouvrage antérieur en disant qu'«il n'est pas question de faire parler un texte muet mais de cerner les particularités de la voix qu'impose sa sémantique. La foi en un discours suppose la perception d'une voix fictive, garant de la présence d'un corps. Une voix qui cependant ne peut avoir qu'une existence paradoxale, puisqu'elle est décalée par rapport au texte qu'elle supporte sans renvoyer à la plénitude d'un corps attesté» (Genèses du discours, Mardaga, i984, p. 98-99). Voir ici même la contribution de 
fiction, une sorte de rêve de transparence, qui tend vers l'autoaffection muette. Se trouvent en tout cas clairement marquées par là les deux bornes entre lesquelles module le ton stendhalien : entre conversation ("écrire comme on parle») qui est un genre à soi seul, et autoconfidence ("écrire presque comme on se parle») qui dessine une sorte d'asymptote de la sincérité. Exprimé autrement : entre le rôle le plus social qui soit, celui de causeur ${ }^{11}$ (dont le modèle pourrait être une sorte de Montaigne "perfectionné " $^{12}$ ?), et l'absence de rôle, entre soi et soi, le lecteur devenant moins qu'un partenaire (qu'il faut distraire, tenir en haleine, surprendre, etc.), mais un autre soimême, idéalement incorporé à l'étoffe du discours qui, dès lors, tend à disparaitre comme moyen entre son émetteur et son récepteur. Le ton, c'est une autre forme de «disparition élocutoire», qui laisse une trace suprêmement personnalisée, feinte et construite, et qui voudrait pourtant se faire oublier comme telle.

Nous croyons que c'est là l'un des aspects essentiels de la question du ton qui, s'inscrivant dans un horizon de sincérité, est de l'ordre du fantasme ou, comme dirait Roland Barthes, d'un imaginaire au second degré. «Faire entendre une voix réelle», selon l'expression de Valéry, c'est forcément créer une illusion - une hallucination. C'est créer une ouïe pour une voix qui n'est pas là - qui n'est là que sous les espèces d'une écriture. Si le ton est une notion intuitive, mal formalisée, qui a tendance à se rabattre souvent sur une sorte de je-ne-sais-quoi, mal vue par conséquent des savants et toujours suspecte, c'est que c'est une notion qui peut à bon droit passer pour «magique» - une in-vocation (qui, comme la voix dont elle est issue ou qu'elle cherche à imiter ou rejoindre, participerait de toute une métaphysique de la présence).

Mais l'illusion n'est pas seulement du côté du lecteur. Valéry saisit immédiatement que le ton n'est pas l'expression d'un caractère, la transmission a-problématique d'un contenu, fût-il psychologique : le caractère n'est pas une denrée solide qu'il suffirait de mettre à la poste de la littérature pour qu'elle soit délivrée en mains propres à son destinataire. Stendhal a son caractère, soit! mais dès qu'il écrit, ce caractère, il se le façonne aussi en écrivant ${ }^{13}$; je reçois le sentiment de ce caractère dans sa prose; c'est ce qui s'appelle le ton.

Fr. Vanoosthuyse sur la voix comme interprétant, à la mode peircienne, plutôt que comme incarnation du texte.

I I. Voir M. Fumaroli, «La conversation», dans Trois Institutions littéraires, Gallimard, «Folio», I992, spécialement p. 202.

I 2. «Le ton de Montaigne fait presque aujourd'hui tout son mérite, perfectionner ce ton. L'amener à la familiarité du meilleur ton possible» (Journal littéraire [juin I 804], t. I, dans Euvres complètes, V. Del Litto éd., Cercle du Bibliophile, I 967-1972, p. 432).

I3. Il importe de rappeler la «définition» du Brulard: «J'appelle caractère d'un homme sa manière habituelle d'aller à la chasse du bonheur.» 
Mais la médiation n'est pas un simple canal. Si le ton se forge dans l'écriture, le caractère également, comme on le notait à l'instant. La sincérité - si sincérité il y a (mais Valéry sait que la sincérité est un «jeu de langage», c'est-à-dire ni un mensonge ni un aveu, mais une opération) - décolle d'elle-même. Valéry dit que Stendhal «imitait à merveille» son propre caractère. C'est dire que la sincérité est une notion «constructiviste» : elle se fabrique grâce et malgré cette médiation obligée de la langue, et c'est sous cette double contrainte que le ton doit s'entendre, non pas comme une émanation que le discours capturerait et qui, comme un parfum, serait libéré à la lecture. La sincérité s'expose, s'exagère, «roque», s'ironise, se singe, etc., bref, elle se joue. Illusion, donc, au sens propre, où la vérité de soi se met en abîme, se risque en acceptant de se traduire, donc de se parjurer, c'est-à-dire de n'être pas transparente, de chatoyer, entre un écrire comme on en parle et un écrire presque comme on se parle, c'est-à-dire comme on se tait. Il y a matière à beaucoup de rhétorique entre ces deux repères.

Ce miroir qui rêve de s'abolir, dans les faits, est condamné à se voir toujours décalé d'avec son reflet. L'ego n'est qu'un précipité ou un résidu de l'égotisme, qui est dénué d'image et se machine selon une ligne de fuite qu'il faut saisir entre présence et absence, comme une proie et son ombre. Imaginaire au second degré, disions-nous? À $n$ degrés plutôt. Le ton est une manière d'arrêter cette récursivité vertigineuse de soi tentant d'embler sur soimême, et ne réussissant le plus souvent qu'à prendre la pose devant soi, de se solidifier en une attitude.

C'est dans cet esprit qu'en traite Valéry. Il voit Stendhal comme un comédien jouant son propre rôle, un rôle aussi proche de lui-même qu'il peut, mais un lui-même idéalisé en quelque sorte, dans le sens de ses vœux, un moi plus rêvé que réel, un moi choisi et quintessencié. Stendhal «se fait» un ton, fondé sur la sincérité et la «vie», mais, au jugement de Valéry, un peu trop vivant justement et affichant un peu trop sa sincérité - «hystérisé», dirait-on aujourd'hui; ou falsifié par la volonté, tel qu'il ne produit, par son excès même, qu'un «effet de comédie», là où il aurait souhaité un simple matter of fact, une innocente transparence qui eût agi par simple exposition (en deçà même de l'«expres$\left.\operatorname{sion}^{14} \gg\right)$. Valéry croit flairer là «le projet d'être soi, d'être vrai jusqu'au faux».

La nudité, l'absence de supplément à la vérité de l'être-soi se voit toujours modifiée par l'éclairage du «théâtre mental». Autant dire qu'elle est rigoureusement impossible. C'est l'éternelle lutte des rhéteurs contre la terreur.

I4. M. Blanchot, qui cite le texte Valéry à propos du ton, fait toutefois plus que lui crédit à la naïveté de Stendhal en distinguant, à propos de la correspondance, les lettres qui «imitent la simplicité», et celles où «être simple n'apparaît point comme le résultat du calcul qui cherche à atteindre la simplicité en montrant ce qu'elle peut être, en exhibant un personnage de comédie» («Stendhal et les âmes sensibles», Faux Pas, Gallimard, I 943, p. 303 ). 
Sceptique jusqu'aux dernières conséquences, Valéry affirmera même que la tricherie qui consiste à «faire vrai» n'est en définitive qu'une variante de celle qui consiste à flatter le modèle. Elle est même peut-être pire en ce sens que plus désespérée.

On peut voir là le deuxième sommet de l'étude de Valéry. Le «faire vrai», qui est en quelque sorte la note de fond du ton, avant toute adjectivation - sorte de degré zéro du mensonge romanesque - la prétention d'être soi-même en se montrant, voire de l'être davantage, de penser complètement absorber toute fictionnalité par le discours, de rendre celui-ci entièrement perméable à la personne, de faire de la médiation non un obstacle, mais un «médium» au sens magique, tout cela renferme un aveu de faiblesse : celui d'une impuissance à exciter l'intérêt du lecteur «par les moyens purement littéraires» (où l'on peut lire la prophétie de l'évolution de la littérature moderne vers l'autofiction). Mais ce n'est pas tant le jugement qui nous intéresse que le fait qu'il pose la question de la sincérité en termes de rôles («il se peuplait l'existence de son mieux») qui ouvre sur une conception théâtrale, dramaturgique de l'énonciation, mais surtout qui, philosophiquement, suppose une ontologie relativiste et graduelle : «le soi a des degrés ${ }^{15} »$. Le monnayage du ton en tons serait, au choix, une issue possible, sous la forme de mise en scène, à l'introuvable unité du moi, une diversion, ou un aveu de la fictionnalité choisie et orchestrée de ce moi, dont l'unité ne s'impose pas moins en dépit du vestiaire d'Arlequin.

Les pistes tracées par Georges Blin et Paul Valéry contiennent suffisamment de stimulations pour remplir l'agenda d'un honnête homme, et presque autant de questions dont il n'est pas certain que plusieurs volontés viennent facilement à bout: nous pensons à l'épineux problème du rapport de la voix au ton par exemple, qui pointe justement vers le défaut de définition sûre de cette notion de ton, définition à laquelle bien peu se risquent ${ }^{16}$. Il en va moins d'une inadvertance ou d'un excès de témérité de la part des utilisateurs que

I 5 . «Le théâtre réfute le schisme absolu entre la parole apprise et la parole inventée, entre le mensonge et la sincérité», montre M. Crouzet (Stendhal et le langage, Gallimard, I 98 I, p. 67).

I6. Dans un article de L'Information grammaticale ("Vers une linguistique des styles», $\mathrm{n}^{\circ} 89$, mars 200I, p. 4), Fr. Rastier définissait les tons (significativement au pluriel, mais la question demeure : le ton est-il la somme ou la synthèse de tons, et laquelle?) : «des isotopies évaluatives [qui] situent les textes dans des dimensions éthiques et pathétiques». M. Parmentier, par exemple, qui assure que la crédibilité morale du narrateur est d'autant plus forte qu'elle est fondée sur plusieurs images narratoriales coexistantes, propose, dans Stendhal stratège. Pour une poétique de la lecture (Droz, 2007), une décomposition en trois tons (qu'elle reprend et développe dans son article ici même) : sentimental, bon ton et «philosophe qui voit de haut». Dans ses Éléments de stylistique française (PUF, I986, p. I 57 et suiv.), G. Molinié inventoriait treize tons (y inclus certaines catégories techniques que D. Combe excluait). 
d'une richesse de la notion et du sentiment d'une connaissance intuitive partagée du mot, d'une évidence sensible telle qu'il deviendrait superflu de le définir. Néanmoins, au seuil de nos travaux, avant d'entrer dans les discussions plus particulières, il importe de fixer quelques idées sommaires par une brève exploration lexicale; nous nous détournerons donc des esprits brillants pour nous adresser aux laborieux dictionnaires.

Que le terme de ton réunisse tout un bouquet d'acceptions explique certainement en grande partie son succès; sa polysémie, se retrouve dans toutes les langues européennes (et bien d'autres, sans doute); elle ne découle pas d'une simple facilité métaphorique, mais relève bien plutôt d'une catachrèse, les extensions de sens étant toutes parfaitement lexicalisées. La plasticité de ton témoigne de ce que le mot cherche à rendre compte d'une réalité de synthèse ou transversale; il possède cette particularité remarquable d'avoir une validité trans-esthétique. Que le ton fasse partie du vocabulaire de base de la peinture, de la musique ou de la littérature ne peut pas être indifférent à sa nature ${ }^{17}$.

Afin de pointer quelques lieux de questionnement, toujours de manière apéritive, on commencerait de démêler la pelote en mettant un peu de simplification : on distinguera ainsi quatre plans - dont on s'apercevra bien vite qu'ils ne sont pas étanches les uns à l'égard des autres, et que le fait même qu'ils communiquent n'est pas non plus étranger à une autre propriété fondamentale du ton : celle de traverser les niveaux de description.

Le premier plan serait celui du corps. Nous ne nous y attarderons pas, sinon pour signaler que le grec tonos signifie «son», «ton», «accent», mais aussi «tendon», «corde», «nerf», le ton étant produit par les vibrations des cordes. C'est la tension artérielle en italien, notre «tonus» (Les cordiaux, disait-on, donnent du ton à l'estomac). En peinture, où il apparaît d'abord, il désigne le degré d'intensité ou d'éclat des teintes. Dans le domaine qui nous occupe plus particulièrement, c'est un terme qui aura à faire avec les questions de tension (narrative), de dynamisme communicatif et, plus largement, avec la partie physique de la substance romanesque et la qualité de l'énergie, dont on sait qu'elle n'est pas chez Stendhal une question subsidiaire ${ }^{18}$.

Nous rencontrons ensuite le ton comme notion «sociologique». Mais on y parvient par degrés insensibles et par un progressif enrichissement de sa valeur physique $^{19}$. La locution à baut ton, par exemple, ne signifie pas autre chose que

17. Cette suspicion à l'égard de la métaphore se retrouve dans le texte d'É. Bordas ou, argumentée différemment, dans celui de Fr. Vanoosthuyse ici même.

I8. On lira ici même la contribution de L. Lassagne.

I9. "L'instance subjective qui se manifeste à travers le discours ne s'y laisse pas concevoir seulement comme statut ou rôle, mais comme "voix", et au-delà, comme "corps énonçant”, historiquement spécifié et inscrit dans une situation que son énonciation tout à la fois 
«à haute voix»; mais, comme si une qualité physique, dès que perçue donc interprétée, prenait des significations autres qu'objectives, qu'elle n'était jamais un pur objet physique mais, comme l'enseigne la psychologie gestaltiste depuis longtemps, acquérait d'emblée des résonances de sentiment ou de sens, le ton désigne une expression. Parler d'un ton de maître, c'est donner à son discours des inflexions qui font entendre une certaine qualité morale, d'autorité, teinte dans la masse, pour ainsi dire; c'est lui conférer certains traits révélateurs d'une disposition psychologique ou d'un statut. Le ton n'est pas appréhendé comme un objet doué de prédicats objectifs puis interprété, mais immédiatement saisi sur fond phorique, définissant, par le contenu qu'il véhicule et contemporain de celui-ci en toutes ses parties, une sorte de moyenne caractéristique, de «bande passante» ou de tessiture, qui renvoie à un type d'expression saisi dans un univers qualifié et du sein d'une interaction, et prenant sens dans un certain habitus moral (au sens large des mours). À l'époque où les mots de thymisme, d'hexis, d'éthos... sont encore barbares (je veux dire grecs), le ton se prête à couvrir ce que la sociologie ou la linguistique désignent aujourd'hui sous ces notions : une façon particulière de s'exprimer et de se présenter, qui, au-delà du langage, touche aux manières en général. On parle du ton de la Cour, de celui des Halles. Le sens de «manière de se comporter dans un groupe social» est attesté vers la fin du XVII ${ }^{e}$ siècle. Le ton est, de la sorte, une forme de réglage de l'expression, une accommodation en fonction du rang, du tempérament, des circonstances, d'un milieu, etc. ${ }^{20}:$ l'un de ses meilleurs équivalents serait peut-être le terme de contenance.

Que le sens moral s'affirme, au détriment du physique, et le domine, le ton désignera alors une modalité du langage, un ensemble de caractéristiques qui typifie la nature du discours en situation et suffit à le faire reconnaitre comme tel : le ton de la flatterie, de la sincérité. Sémiotiquement manifesté, le ton suppose alors un déchiffrement, une interprétation morale.

C'est cette articulation qui reste mystérieuse, précisément, celle du matériel (les caractéristiques du discours) et de l'impression subjective (la saisie d'une unité tonale), dont on se doute qu'elle repose sur des «compétences

présuppose et valide progressivement» (D. Maingueneau, «Éthos, scénographie, incorporation", op. cit., p. 76).

20. Voir Marmontel, dans ses Éléments de littérature (1787) : «Le langage a différents tons, celui du bas peuple, celui du peuple cultivé, celui du monde et de la cour, qu'on appelle familier noble, celui de la haute éloquence, celui de la poésie héroïque, et dans tout cela une infinité de gradations et de nuances qui varient encore selon les âges, les conditions et les mœurs. [...] Les tons différents dont je parle sont à la langue ce que les divers modes sont à la musique [...] chaque style a de même un cercle de mots, de tours, et de figures qui lui conviennent, et dont plusieurs ne conviennent qu'à lui. C'est dans ce cercle que la plume de l'écrivain doit s'exercer» (S. Le Ménahèze éd., Desjonquères, 2005, article «Analogie du style»). 
endo-narratives ${ }^{21} \gg$ (on n'a rien inventé de tel que les termes techniques pour escamoter un problème), qui empruntent à une phénoménologie plus ou moins implicite du faire, à nos expériences, nos répertoires, nos habitudes praxiques, ce que nous n'apprenons pas dans les textes, mais par familiarité avec ce vaste domaine que Ricœur nomme la «préfiguration ${ }^{22}$ ». Les textes, à leur tour, et par rétroaction, perfectionnent, enrichissent, dialectisent, mettent en perspective, etc., notre perception et notre sentiment d'un monde où être, faire et dire forment un tout indémêlable.

Que de descriptif, il se fasse normatif, et il faudra entendre le ton, absolument, comme bon ton, soit : les manières convenues dans le «meilleur» milieu, le monde poli, élégant, valorisé et donné comme modèle ${ }^{23}$. Se mettre au ton, dira-t-on par exemple, est chose qui répugne aux originaux. Ironie de l'évolution lexicale et paradoxe social à la fois : le ton se retrouve ici aux antipodes de ce qu'il sera réputé être bientôt : non pas une marque idiosyncrasique, mais un conformisme, une absence d'originalité. La contenance de tantôt s'est figée en convenance.

La rhétorique ne représente ici qu'un cas particulier d'un phénomène plus général, qui touche aux mœurs. Le ton, comme norme, n'est que la convenance du style à la nature du sujet, dira Buffon dans son Discours de Réception, et, dans l'article «style» de l'Encyclopédie où sont énumérées de manière très précise les propriétés qu'il en exige, on lira, aux côtés de la propriété des termes, du tour, des coloris, des sons, des traits et de la manière, la «propriété du ton», citée en deuxième place, définie comme l'«assortiment du style au genre $\left.{ }^{24}\right\rangle$. Double assujettissement : au contenu ou à la visée du propos d'une part, au milieu d'émission et de destination d'autre part. «Nature du sujet» ou «genre» sont les régulateurs du ton, réduit au rôle d'une sorte de variable d'ajustement, d'adaptation de la manière du discours aux nécessités de son énonciation, où ne s'exalte pas mais bien plutôt s'oblitère le caractère personnel, au service du decorum. Quand il n'achève pas de se confondre purement avec les vocables de genre ou de style : le ton oratoire, pathétique... On n'entend point dans un sermon des «tons de théâtre», note La Bruyère comme un précepte; «C’est

21. Voir B. Gervais, Récits et actions. Pour une théorie de la lecture, Les éditions du Préambule, Québec, I990.

22. À la réserve près que cette «préfiguration» ne concernerait la diégèse qu'en tant qu'elle est affectée d'un certain coefficient de narration (telling) : la logique des actions, mais aussi l'entente des affects, les schémas mais aussi les modes, l'agir mais aussi le pâtir.

23. Lire l'article «Bon ton» d'Y. Ansel dans le Dictionnaire de Stendhal, Champion, 2003.

24. Diderot et d'Alembert, Encyclopédie, ou Dictionnaire raisonné des sciences, des arts et des métiers, Paris, Briasson, I75I-I 780, t. XV : p. 24I-242. Voir J.-Ph. Saint-Gérand, «Introduction. Contextualisations épilinguistiques. À propos de Stendhal (I 783-I 842)», dans Ph. Berthier et É. Bordas éd., Stendhal et le style, Presses Sorbonne Nouvelle, 2005, p. 26. 
un grand talent que de prendre toujours le ton convenable au sujet qu'on a à traiter», confirme Grimm.

Nous sommes ici en présence de déterminations qu'on dira extrinsèques au ton. Mais au XVII ${ }^{\mathrm{e}}$ siècle (on voit que les plans que nous avons dégagés pour la présentation ne sont pas sagement successifs), ton prend le sens étendu de «manière de s'exprimer par écrit», et particulièrement en parlant du style caractéristique d'une œuvre littéraire. On reconnaitra sans peine le plan de l'elocutio; le ton acquiert ses lettres de littérature et s'approche pour de bon dangereusement de la notion de style. Tout en conservant le contact avec l'oral, cependant $\mathrm{t}^{25}$. C'est un autre point, de grande importance croyons-nous, à l'origine d'une possible démarcation style/ton, que ce dernier, désormais pleinement transposé à l'écrit, n'en conserve pas moins quelque chose de sa source orale ${ }^{26}$; le ton est une manière de s'adresser à quelqu'un : il n'est jamais totalement séparé d'une attitude, voire d'une pose. Le prendre sur un certain ton est glosé dans le dictionnaire par : avoir une certaine conduite, un certain langage. Voilà encore un nouement possible du complexe du ton : celui du langage et de la conduite. Le ton, c'est du langage, mais pris dans la situation, les codes de civilité, les notions de décence, de norme, la posture, etc., et, last but not least, d'un langage qui engage une interlocution ou, plus largement, une forme d'interaction, donc une prise en compte du public ${ }^{27}$. Se mettre au ton de quelqu'un, c'est adopter ses idées, ses manières, son langage (on parlerait peut-être aujourd'hui de recherche d'empathie), c'est se «syntoniser», «tonaliser» son discours pour l'adapter à l'usage qu'on en veut faire (on se souvient que Stendhal confiait à Balzac "prendre le ton» de La Chartreuse en lisant le Code civil), pour faire entendre un certain mode sous-tendant le dire, et atteindre celui avec lequel

25. Cela correspond - si l'on veut éviter de faire servir les mêmes mots à toute main, et employer une terminologie plus technique par conséquent - à ce que D. Maingueneau a plus précisément désigné sous le terme de «scénographie», choisissant le mot à dessein pour conjoindre à la dimension théâtrale de la «scène» celle de la -graphie, de l'«inscription».

26. Pour être correctement interprété, le texte écrit, doit, selon D. Anzieu, contenir, outre un respect du code langagier en vigueur, «des modalités de l'énonciation, qui sont des équivalents stylistiques de l'intonation et du geste» et rendent présent le corps des interlocuteurs. Assimilant purement et simplement ton et style, il fait de ce dernier, à l'écrit, le transposeur des «contiguités sensorielles et motrices» de l'oral, ce qui est communiqué du sens dans «l'accompagnement vocal ou gestuel, par une contagion tonico-posturale, par une empathie rythmique». (Le Corps de l'cuvre, Gallimard, I98 I, p. I72-173, I 80). Cet aspect de transposition du corps avait été bien vu encore, dès I 930, par Volochinov; voir «Des frontières entre poétique et linguistique», traduit par G. Philippenko et M. Canto, dans T. Todorov, op. cit., notamment p. 270 et suiv.

27. Non seulement le style peut avoir, mais «il doit avoir, plusieurs tons, relativement aux sujets que l'on traite et aux personnages qu'on fait parler $[. .$.$] . Ces règles de convenance [. .$. s'étendent jusqu'aux personnes qu'on a dessein d'intéresser ou de persuader en écrivant» (Marmontel, op. cit., p. 1075-1076). 
on souhaite s'accorder; dans tous les cas, cela traduit moins un état qu'un procès soucieux de prendre constamment en considération ces contraintes.

Le mot rejoint alors les valeurs de la très riche Stimmung, qui en constitue une possible traduction, puisque la Stimmung est une manière de s'accorder affectivement ${ }^{28}$. Le mot allemand, avec tous ses arrière-plans philosophiques et l'usage qui en a été fait en phénoménologie, se déploie dans plusieurs directions, réunissant les sèmes d'humeur, d'état d'âme, mais aussi de justesse, de tendance, etc. ${ }^{29} \mathrm{Il}$ suppose plus qu'une conformation : une certaine disposition modale du discours (en rapport avec un destinataire) mettant en jeu l'affect, l'énergie, une orientation générale qui ploie l'ensemble du discours selon un certain geste (comme les champs sous le pinceau de Van Gogh). Une phrase de Fontenelle le dit naïvement : «La géométrie n’a qu'un ton; mais peut-être ferait-elle bien d'en changer quelquefois un peu, puisqu'elle parle à des hommes.» Prenant en compte un destinataire, le ton commence comme un phénomène physique, on l'a vu, et aboutit à un phénomène physique en ce qu'il est fait pour toucher quelqu'un, formant ainsi une sorte de boucle dont la matière est l'expression : «Mes pauvres lettres n'ont de prix que celui que vous y donnez en les lisant comme vous faites, car elles ont des tons, proteste Mme de Sévigné à sa fille, et ne sont pas supportables quand elles sont ânonnées ou épelées »- où l'on retrouve le vif du ton sous et par l'écrit, quand celui-ci se montre apte à porter en lui une diction ${ }^{30}$.

Ce dernier exemple prouve ce qui est une évidence : que le départ n'est jamais absolu entre fond et forme, que le ton, en l'espèce, n'est pas un simple «habillage» du discours, sa couche superficielle, son vernis (n'en déplaise à Stendhal! $)^{31}$, mais concerne «la coloration axiologique de la totalité du matériau

28. J.-M. Schaeffer note que «nous sommes démunis pour penser tout ce qui relève de la Stimmung (de la tonalité) des choses plutôt que de leur Bestimmung (de leur détermination), donc tout ce qui traduit notre Gestimmtheit, notre façon d'être accordé ou désaccordé au réel» (La Fin de l'exception bumaine, Gallimard, 2007, p. 38).

29. Heidegger fait de l'affection (Befindlichkeit) ou de la tonalité affective (Stimmung) la présupposition de tout comprendre. Chez Maldiney où elle joue un rôle capital, cette Stimmung désigne, antérieurement à la scission extérieur/intérieur, la tonalité d'une situation et des comportements fondés en elle. Au-dessous de l'expérience structurée en Sujet/Objet, elle est, "moment inobjectif des formes», ce mode pathique de l'être-avec-le-monde, sorte de basse continue qui décide du style de présence au monde (comparable, sinon identifiable à l'ethos tel que nous l'avons entendu, ou à la phorie greimassienne), royaume du rythme et du hiéroglyphique. Voir la stylistique d'E. Staiger, et, sur les rapports de la Stimmung avec le style, Y. Baudelle, «Sur les tonalités littéraires : contribution à une poétique phénoménologique», Littérature, $\mathrm{n}^{\circ} \mathrm{I}_{32}$, Larousse, décembre 2003, p. 85-99.

30. Comme le souligne opportunément Fr. Vanoosthuyse dans sa contribution, le ton est celui $d u$ texte et celui que le lecteur adopte : négociation toujours à refaire.

3I. D. Combe distinguait les genres constitués ou proprement dits (subsumés, dans l'esthétique de Goethe et Schiller, par des «manières de sentir» ou Empfindungsarten) et les 
verbal» (Volochinov). L'une des finalités d'une étude théoriquement approfondie du ton pourrait même consister à se demander comment repenser cette schize fond/forme que trop de conceptions du style ont tendance à aggraver en autonomisant cette notion de style. Quand Littré parle d'être sur un ton, il s'agit bien de dire des choses d'une certaine espèce, ayant trait à un certain sujet, ou propos. «Pendant que nous sommes sur ce ton-là $[\ldots]$ », commence pareillement Mme de Sévigné, faisant allusion aux intrigues amoureuses de la cour d'Angleterre, donc à un «contenu» également. Plusieurs dictionnaires citent proverbialement: C'est le ton qui fait la musique ou la chanson, pour signifier que la manière dont on dit les choses dénote l'intention véritable de celui qui les dit. Où l'on vérifie une fois de plus que le ton est adjectif et substance à la fois, matière et manière conjointement. Oswald Ducrot - si nous nous transportons par un raccourci jusqu'à aujourd'hui - rattache de même l'ethos (dont on peut considérer qu'il est l'un des principaux candidats à prétendre ramasser la mise et reprendre l'héritage du ton dans la linguistique et la rhétorique contemporaines $^{32}$ ) tant à l'inventio (le choix des arguments) qu'à l'elocutio (le choix de la mise en mots) et à l'actio (mise en voix, gestes, ton) ${ }^{33}$.

Avec notre quatrième et dernier plan, la notion de ton relève moins d'une définition positive qu'elle n'indique une limite de la rhétorique. Pour citer encore Mme de Sévigné, cette éloquente épanorthose : «De quel ton, de quel cœur, car les tons viennent du cœur $[\ldots]$ ». Le ton entendu ainsi nous fait sortir du cadre de la seule linguistique; il n'est pas un terme technique, il ne relève plus de protocoles rhétoriques, mais montrerait plutôt la limite de cette discipline, et cet usage non technique demande à être interrogé sinon comme la «vérité» de la notion, du moins comme son point de fuite. Mais c'est de l'ensemble de ces quelques sondages rapides qu'il ressort, semble-t-il, une «compréhensivité» certaine des acceptions du terme, qui confirme que le

catégories (ou qualités) génériques : «tonalités affectives» (lyrique, dramatique, etc.) - à distinguer des «catégories techniques» (le descriptif, par exemple) qui échapperaient aux tonalités (Les Genres littéraires, Hachette, i 992, p. I7-2I). Ici même, par exemple, D. Sangsue et G. Mathieu illustrent ce que je nommerais le ton comme veine, celle du fantastique et celle du pornographique. G. Molinié, qui reconnaît que le ton est «la question la plus vaste» en stylistique, privilégie, sur les genres (substantifs), ces «inflexions tonales» (adjectifs substantivés), qui les débordent et les transcendent (La Stylistique, PUF, $3^{\mathrm{e}}$ éd., 200I).

32. D. Maingueneau, par exemple, relie la notion d'éthos «à la notion de ton, qui relaie avantageusement celle de voix dans la mesure où elle renvoie aussi bien à l'écrit qu'au parlé. Le ton s'appuie à son tour sur une «double figure de l'énonciateur, celle d'un caractère et d'une corporalité» (Genèses du discours, op. cit., p. Ioo). De même, côté réception, ce qui compte "ce n'est point tant le résultat sonore, mais l'orientation qui rend possible l'intonation" (V. N. Volochinov, «Les frontières entre poétique et linguistique», op. cit., p. 280).

33. Le ton, dernier cité, dont l'éthos a repris la généralité, n'est plus ici entendu que comme un facteur lié à la seule performance orale. 
ton est un phénomène sinon «total», du moins non local (d'où sa prédisposition à être étudié par la Psychologie de la forme, notamment à travers son concept d'appréhension globale, ou Übersummativität).

Il faut ajouter que le ton se situe entre puissance et actualité; c'est un principe séminal en ce qu'il se «réalise» physiquement, mais qu'il subsume, comme en musique, ce qui décide d'une certaine unité de la pièce du point de vue de sa configuration harmonique (dans laquelle vont s'inscrire les déterminations mélodiques, de timbre...); il est donc toujours présent, sous-jacent, tacite, dans l'expression, partout agissant même et surtout en dehors de ses saillances ${ }^{34}$. D'où la question que les poétiques du continu peuvent légitimement se poser : Est-ce encore le ton que l'on saisit quand on le détaille, quand on en démembre les composantes? quelle réalité possède-t-il en dehors de son mode d'être continu?

La musique ici peut être une source d'inspiration méthodologique et l'étude de la tonalité, avec les précautions d'usage, trouverait des analogies naturelles avec le ton dans le domaine de la littérature. Celui-ci ne pourrait-il être ainsi envisagé comme le nom d'un système d'intervalles fondamentaux qui, comme une échelle de sons, constitue une réalité substantielle, mais aussi différentielle avant tout? La tonalité organise en effet le rapport entre les éléments d'un tout, en fonction d'une gamme (dont le genre fournirait l'équivalent en littérature?); elle n'est pas une collection de phénomènes isolés, hétérogènes, mais un organon hiérarchique, qui possède ses échelles, ses attractions, ses harmoniques et ses tendances résolutives privilégiées. On peut imaginer que le ton en littérature définisse lui aussi son champ de gravitation et s'organise en fonction d'un son principal, sa tonique, désignée en musique par un nom, la majeur, par exemple. Reste à définir ce qui, pour chaque œuvre, jouerait le rôle de ce «son principal ${ }^{35} \gg \ldots$

Nous ne retarderons pas davantage le moment d'entrer dans les études de détail, mais nous souhaiterions avant de céder la parole indiquer au moins l'une des conséquences de la réflexion sur le ton chez Stendhal, et l'un de ses enjeux. Il nous paraît qu'elle donne à réfléchir, par contraste, sur la recherche d'«atonie» d'un Flaubert par exemple ${ }^{36}$. La soustraction forcenée de la voix,

34. À propos des rapports entre musique (Tonkunst, Tonspiel) et langage, Kant avance : «toute expression du langage possède dans un contexte un ton, qui est approprié à son sens; ce ton indique plus ou moins une affection du sujet parlant et la provoque aussi chez l'auditeur, et cette affection éveille l’idée en celui-ci, qui est exprimée par un tel ton dans la langue; la modulation est donc en quelque sorte une langue universelle des sensations, intelligible à tout homme, que la musique seule emploie dans toute sa force» (Critique de la faculté de juger, $\int 53$, trad. A. Philonenko, Vrin, I986).

35. É. Bordas a son idée sur la question : on lira son article ici même.

36. Le parallèle est presque aussi classique que celui qui oppose Corneille à Racine! On le trouve chez Alain, par exemple, qui parlait de ses "préférés», Stendhal et Balzac, qui 
résorbée dans la diction, est tout à fait différente (et, à bien des égards, le contraire de l'idéal du gueuloir, dont on nous a rebattu les oreilles : laboratoire non de la parole vive, mais plutôt de la déclamation $\left.{ }^{37}\right)$. Que Flaubert soit devenu comme l'emblème même de la littérature telle que la modernité enfin l'a révélée à elle-même, une littérature comprise et revendiquée comme coupée d'avec toute interlocution, et qui même se rêve coupée de toute énonciation ${ }^{38}$, se tenant toute seule, comme un dolmen, sans auteur, blanche (et, votivement, sans lecteur?) peut nous faire nous interroger sur cette anachronique notion de ton, qui est contradictoire avec l'effacement du narrateur, mais aussi avec un narrateur conçu essentiellement comme rhéteur. Flaubert est l'un des principaux artisans d'une littérature voulue sans personnalité, dépourvue de cette dimension de l'bypocrisis (que les Latins traduisaient par actio) : plus de jeu, plus de «comédie». C'est l'esprit de sérieux qui s'empare du roman, et la «littérature» qui se distingue résolument de la fiction. "Il est trop particulièrement soi, dit Valéry de Stendhal, pour être réductible à un écrivain.»

Dans ce débat, il ne s'agit pas seulement d'une affaire d'«énonciation», mais de rien de moins que de la question de la présence-de-quelqu'un dans le discours, contre laquelle toute une tradition nous a appris à nous tenir en garde. Dans le même volume de Variété, Valéry notait combien l'opposition «entre le dogme même du Réalisme - l'attention au banal - et la volonté d'exister en tant qu'exception et personnalité précieuse eut pour effet d'exciter les réalistes au soin et aux recherches du style ${ }^{39}$ ». Le style, comme phénomène de compensation de «réalité». Ce qu'il nous intéresse de pointer simplement ici, et ceci nous servira de non-conclusion, c'est que les rapports que nous cherchions à penser, en commençant, du style et du ton, plutôt sous le régime de la complémentarité, se trouvent ici dans une relation de disjonction, voire d'exclusion. Style ou Ton (Flaubert ou Stendhal, pour parler en noms propres). Qu'est-ce

«passent pour n'avoir pas de style; au contraire on en reconnaît en Flaubert, où je n'ai pas trouvé grand-chose» (Propos, II, S. S. de Sacy éd., Gallimard, «Bibliothèque de la Pléiade», I 970 , p. 459).

37. Évoquant La Dialectique de Ramus, R. Sabry affirme que l'exigence absolue «du lisse et de l'homogène, principalement dans le genre narratif » aboutit à la théorisation d'un récit pur de toutes les scories de ce que Benveniste appellera «discours», décrites en termes d'excès par rapport au degré zéro obligé de la narration, où l'auteur observe une attitude de total désintéressement et de retrait. À la méthode d'art, qui procède comme si elle avait affaire à une écoute abstraite et neutre, s'oppose la méthode de prudence, qui se fonde dans le rapport à une écoute en puissance (Stratégies discursives. Digression, Transition, Suspens, Éditions de l'EHESS, I 992 , p. 7I-75).

38. Privée d'accentuation axiologique - c'est-à-dire de ton, dans le vocabulaire de Volochinov la littérature n'édifie que des «monuments linguistiques pétrifiés» (op. cit., p. 285).

39. P. Valéry, op. cit., p. 6 I 4. 
que chacune des branches de cette alternative engage? ce serait l'une de nos interrogations, au seuil de notre enquête à plusieurs détectives.

Nous remercions, en la personne de sa directrice, Marie-Rose Corredor, le Centre d'études stendhaliennes et romantiques de l'université StendhalGrenoble 3 - cadre de la journée d'étude (juin 2007) qui fut la première étape de ce travail - ainsi que Bertrand Vibert et Claude Coste, qui ont bien voulu accueillir sa version définitive dans la revue qu'ils dirigent. 